\title{
Nuevos desafíos en cirugía fetal
}

\author{
FERNANDO VULETIN S.
}

1. Sección de Cirugía Pediátrica, División de Cirugía, Escuela de Medicina. Pontificia Universidad Católica de Chile.

\begin{abstract}
\section{Current trends in fetal surgery}

The advances in prenatal diagnosis have allowed understand with better precision the fetal physiopathology and recognize the diseases that are life-threatening. Most prenatally diagnosed anatomic abnormalities are best treated after birth, but a small number of malformations than can cause fetal demise or devastating consequence postnatally, may be corrected before birth. The concept of fetal surgery was conceived in order to prevent fetal or early postnatal death, or to prevent irreversible organ damage. Because most of these surgical procedures involve some risk to the fetus and the mother, the surgery must be safe and effective. This requires knowing information about the physiopathology and natural history of the disease, the efficacy of the surgical intervention to ameliorating the disease and the safety of the planned intervention, all this primarily tested on animal model. The purpose of this article is to describe the current status of fetal surgery, with focus on the most commonly congenital anomalies treated by open fetal surgery.
\end{abstract}

(Key words: Fetal surgery, prenatal diagnosis, congenital malformations, newborn).

Rev Chil Pediatr 2013; 84 (3): 254-261

\section{RESUMEN}

Los avances en el diagnóstico prenatal han permitido comprender con mayor precisión la fisiopatología fetal y reconocer las enfermedad que ponen en riesgo su vida. La mayoría de las anormalidad anatómicas diagnosticadas prenatalmente, son mejor tratadas después de nacer, pero existe un pequeño número de malformaciones que puede causar la muerte del feto, o provocar consecuencias devastadoras postnatales, y que pueden ser corregidas antes de nacer. El concepto de cirugía fetal fue concebido en orden de prevenir la muerte fetal o postnatal temprana, o para prevenir el daño irreversible de órganos. Como la mayoría de estos procedimientos quirúrgico envuelven algún riesgo al feto o a la madre, la cirugía tiene que ser segura y efectiva. Esto requiere saber información acerca la fisiopatología e historia natural de la enfermedad, la eficacia de la intervención quirúrgica y la seguridad de la intervención programada, todo esto primariamente probado en modelos animales. El propósito de este artículo es describir el estado de la cirugía fetal, con foco en las anomalías congénitas que más comúnmente se están tratando mediante cirugía fetal abierta.

(Palabras clave: Cirugía fetal, diagnóstico prenatal, malformaciones congénitas, recién nacido).

Rev Chil Pediatr 2013; 84 (3): 254-261

Recibido el 12 de abril de 2013, aceptado para publicación el 15 de mayo de 2013.

Este trabajo cumple con los requisitos sobre consentimiento /asentimiento informado, comité de ética, financiamiento, estudios animales y sobre la ausencia de conflictos de intereses según corresponda.

Correspondencia a:

Dr. Fernando Vuletin Solís

E-mail: fvuletin@med.puc.cl 


\section{Introducción}

Con los avances en el diagnóstico prenatal, ha surgido una nueva población de pacientes que ha enfrentado a obstetras, neonatólogos y cirujanos pediatras a nuevos desafíos en el manejo de fetos con malformaciones congénitas con riesgo vital.

Sin embargo, los avances en el desarrollo del diagnóstico prenatal, no ha sido seguido de la mano de mejoras en la morbilidad y mortalidad global de estos pacientes, ya que algunos llegan muy enfermos antes de ser poder ser tratados en el período post natal inmediato. Esta situación ha llevado a un grupo de especialistas al desarrollo de la terapia fetal.

Las anomalías detectadas antenatalmente, en la mayoría de los casos, puede esperar para su tratamiento después de nacer. Sin embargo, un grupo de fetos requerirá de una intervención mientras aún está en el útero. Esto puede ser para salvar la vida del feto o para prevenir el daño permanente de órganos. Esta intervención puede corregir la malformación, detener la progresión de la enfermedad o para tratar un situación puntual que pone en riego de vida al feto. La intervención fetal se ha transformado en una opción importante para fetos que de otra manera no sobrevivirán durante el embarazo o que tendrían un significante morbilidad y mortalidad después de nacer.

La primera cirugía materno-fetal abierta reportada fue en 1982 por el Dr. Harrison ${ }^{1}$. Se creó una vesicostomía en un feto con hidronefrosis congénita bilateral. Desde ese momento el campo de la terapia fetal ha ganado importancia y la consideración del feto como un paciente.

Los criterios para realizar cirugía fetal fueron establecidos en 1982 por los fundadores de la Sociedad Internacional de medicina fetal y cirugía $^{2}$ (IFMSS por su sigla en inglés) y siguen estando válidos hasta el día de hoy (tabla 1).

Las anomalías congénitas que cumplen con los criterios para considerar intervenciones fetales se resumen en la tabla 2.

Se discutirán algunas patologías que afectan al feto y procedimientos que han sido demostrado ser útiles en el manejo antenatal de estas condiciones.

\section{Lesiones pulmonares fetales}

Los avances en la tecnología en los equipos de ecografía prenatal han resultado en un aumento en la capacidad de diagnóstico prenatal de la lesiones pulmonares fetales, la mayoría diagnosticada entre las 18 y 20 semanas de embarazo.

Las lesiones pulmonares congénitas incluyen un amplio espectro. El diagnóstico diferencial de estas incluye: malformaciones congénitas adenomatoideas pulmonares o CPAM por su sigla en inglés, secuestros broncopulmonares, enfisemas lobares congénitos y quistes broncogénicos, entre otros ${ }^{3}$. La ecografía nos dará el detalle del volumen de la lesión,

Tabla 1. Criterios para realizar cirugía fetal

1. La habilidad para poder establecer un diagnóstico prenatal exacto

2. Historia natural de la enfermedad bien documentado y pronóstico establecido

3. La presencia de una lesión corregible, la cual si no es tratada, llevará a la muerte fetal, disfunción orgánica irreversible antes de nacer, o morbilidad postnatal severa

4. Ausencia de otras anomalías severas

5. Un modelo animal para la enfermedad y procedimiento debe existir

6. El procedimiento debe ser realizado por un equipo multidiciplinario con protocolos estrictos y aprobado por el comité de ética institucional

7. La familia debe estar aconsejada sobre los riesgos y beneficios del procedimiento. y debe estar de acuerdo con que se realice

8. Los riegos maternos deben ser menores y aceptables

Tabla 2. Malformaciones congénitas actualmente tratadas con cirugía fetal

Lesiones pulmonares fetales

Mielomeningocele

Teratomas sacrocoxigeos

Hernias diafragmáticas congénitas

Síndrome de transfusión feto-fetal

Compresiones extrínsecas e intrínsecas de la vía aérea

Síndrome de banda amniótica

Malformaciones cardiacas

Obstrucciones del tracto urinario bajo 
consistencia, localización, irrigación arterial y drenaje venoso, ayudando a definir el diagnóstico.

La CPAM es una lesión benigna sólidoquística, generalmente restringida a un lóbulo del pulmón. La masa contiene quistes que varían entre menores de $1 \mathrm{~mm}$ a mayores de 10 $\mathrm{cm}$. Histológicamente se caracterizan por un sobrecreciento de los bronquiolos respiratorios terminales. Su irrigación proviene del sistema pulmonar y están conectados al árbol traqueobronquial $^{4}$.

Los secuestros broncopulmonares son una masa de pulmón no funcionante. Se origina de una profusión anormal del intestino primitivo y su irrigación proviene de la circulación sistémica. Existen dos tipos, intralobares y extralobares, dependiendo si están o no incorporados al parénquima pulmonar repectivamente ${ }^{4}$.

La mayoría de estas lesiones tiene un pronóstico favorable sin intervenciones prenatales y la regresión del tamaño de la lesión es comúnmente observado. El pick de crecimiento para estas lesiones está entre las semanas 25 a 28 de gestación, con una regresión espontánea que varía entre $15-65 \%$ para las malformaciones congénitas adenomatoideas pulmonares y hasta $68 \%$ para los secuestros broncopulmonares $^{5}$. Sin embargo, aún con esta regresión en el tamaño y la pérdida de ecogenidad de la lesión, se deben estudiar con tomografía computada todos estos pacientes en el periodo postnatal ${ }^{6}$.

Alguna de estas lesiones puede seguir creciendo a un ritmo no predecible, produciendo alteraciones en el feto secundarias al efecto de masa en el pulmón y estructuras mediastínicas. Compresión del esófago e interferencia con la deglución puede producir polihidroamnios. Compresión del pulmón puede producir hipoplasia pulmonar. La obstrucción de la vena cava puede alterar el retorno venosos y puede desencadenar falla cardiaca y esto llevar al desarrollo de hidrops fetal no inmune ${ }^{7,8}$.

Para evaluar el crecimiento y pronóstico de estas lesiones, Crombleholme ${ }^{9}$ desarrolló una medición que se deriva del cálculo del volumen de la masa pulmonar (largo $\mathrm{x}$ ancho $\mathrm{x}$ alto $\mathrm{x} 0,52$ ) dividido por la circunferencia craneana, el CCAM volumen ratio (CVR). Un CVR $>1,6$ está asociado con una probabilidad de desarrollo de hidrops en un $75 \%$, mientras un CVR $<1,6$ tiene baja probabilidad de desarrollo de hidrops no inmune.

Ecográficamente, en el periodo fetal, estas lesiones se pueden dividir en microquisticas o macroquisticas, dependiendo si los quistes son menores o mayores de $5 \mathrm{~mm}^{10}$. Esta clasificación se correlaciona poco con el tipo histológico post natal, pero ayuda en la evaluación y tratamiento de los casos detectados en útero. Cuando la lesión es predominantemente microquistica y grande, son frecuentemente asociadas a desviación del mediastino, hipoplasia pulmonar, polihidroamnios y desarrollo de hidrops no inmune, requiriendo cirugía fetal abierta para evitar la muerte del feto. Las lesiones macroquisticas tienden a tener mejor pronóstico, ya que tienden a crecer menos rápido, pero cuando el quiste predominante es muy grande, puede requerir intervención antenatal.

La mayor experiencia con lesiones congénitas prenatales pulmonares ha sido reportada por Adzick ${ }^{11}$. Ciento setenta y cinco lesiones fueron encontradas en 12 años. Ciento treinta y cuatro fueron malformaciones congénitas adenomatoideas pulmonares. Catorce embarazos fueron terminados. Ciento un casos fueron manejados expectantemente. Trece pacientes fueron sometidos a cirugía fetal abierta entre las 21 y 29 semanas de gestación con 8 fetos que continuaron la gestación y se resolvió el hidrops entre 1 y 2 semanas después de la cirugía. Seis fetos fueron tratados con drenajes toracoamnioticos. Setenta y seis fetos que no desarrollaron hidrops no inmune tuvieron $100 \%$ de sobrevida. En 25 fetos con lesiones grandes que se complicaros con hidrops progesivo, fueron manejadas expectantemente. Estos fetos tuvieron $100 \%$ mortalidad, ya sea en útero o inmediatamente después de nacer.

El manejo de las malformaciones pulmonares fetales, dependerá del CVR que es obtenido al momento de la presentación. Si el CVR es menor de 1,6 y no hay evidencia de un quiste dominante, la probabilidad de desarrollar hidrops no inmune es menor al 2\%. Si el CVR es menor de 1,6 pero existe un quiste grande, el fetos aun tiene riesgo de crecimiento agudo de la lesión y posterior desarrollo de hidrops. En estos casos la colocación de un drenaje to- 
racoamniotico debería considerarse cuando se observaran mínimos signos de hidrops. Si el CVR es mayor de 1,6 al momento de la presentación, existe un $80 \%$ de probabilidad de desarrollar hidrops no inmune. Una ecografía dos veces a la semana se debería realizar para detectar precozmente los signos de hidrops ${ }^{3}$. La administración de esteroides maternos se debería administrar en todos los casos de CVR mayor de 1,6 , ya que se ha observado que los esteroides podrían frenar el crecimiento de la malformación, llegando a la meseta de crecimiento antes ${ }^{12}$.

Los fetos con lesiones con un quiste dominante en los cuales se desarrola hidrops antes de las 32 semanas de gestación, pueden ser consideradas para tratamiento en útero con drenajes toracoamnióticos, con resultados que alcanzan el $70 \%$ de sobrevida ${ }^{13}$. En los casos de lesiones microquisticas grandes con desarrollo de hidrops antes de las 32 semanas, y que no mejoran con la administración materna de esteroides, se debería considerar toracotomía fetal para resección de la lesión (figura 1). Los series reportadas muestran resultados de sobrevida que alcanzan aproximadamente el $50 \%{ }^{4}$. En casos de fetos con lesiones que producen hidrops y que las gestaciones son mayores de 32 semanas, se podría considerar la realización de la resección de la lesión por medio de una toracotomía mientras en feto está conectado a la circulación placentaria, lo que se denomina EXIT a resección ${ }^{14}$. De esta manera el feto que tiene su mediastino desplazado y comprimido, se evitará los problemas ventilatorios y circulatorios que se presentarían si se intentara realizar la cirugía una vez nacido.

\section{Mielomeningocele}

El mielomeningocele (MMC) o espina bífida, es una defecto congénito del sistema nervioso central en el cual existe una profusión de las meninges y elementos neurales a través de una abertura en los arcos vertebrales. Está asociado con un grado variable de parálisis de las extremidades inferiores, retraso en el desarrollo y alteración en la función intestinal y vesi$\mathrm{cal}^{15}$. Además, los pacientes con MMC desarrollan la malformación de Arnold-Chiari tipo 2 , que consiste en desplazamiento caudal de los elementos de la fosa posterior a través del foramen magnun, resultando en desplazamiento inferior del vermis cerebelar, elongación y desplazamiento de la médula y compresión del tronco cerebral. Esto lleva a una circulación anormal del líquido cerebroespinal, resultando en la aparición de hidrocefalia que requiere la colocación de drenajes ventriculares en el 80 a $90 \%$ de los $\operatorname{casos}^{16}$. La colocación de drenajes ventriculares resulta en un morbilidad significativa, con cerca del $64 \%{ }^{17}$ de los pacientes presentando obstrucción o infección en el primer año post instalación y $95 \%{ }^{18}$ requerirá una revisión durante los primeros 25 años de vida. La malformación de Arnold-Chiari tipo 2 está asociada además con disfunción del tronco cerebral, incluyendo apneas centrales, dificultades en la deglución y disfunción de los nervios craneales, representando la principal causa de muerte en los primeros 5 años de vida ${ }^{18}$.

Existe la hipótesis propuesta de los "dos golpes" para explicar la fisiopatología del $\mathrm{MMC}^{19}$. El primero estaría dado por la falla en el cierre del tubo neural con la consecuente

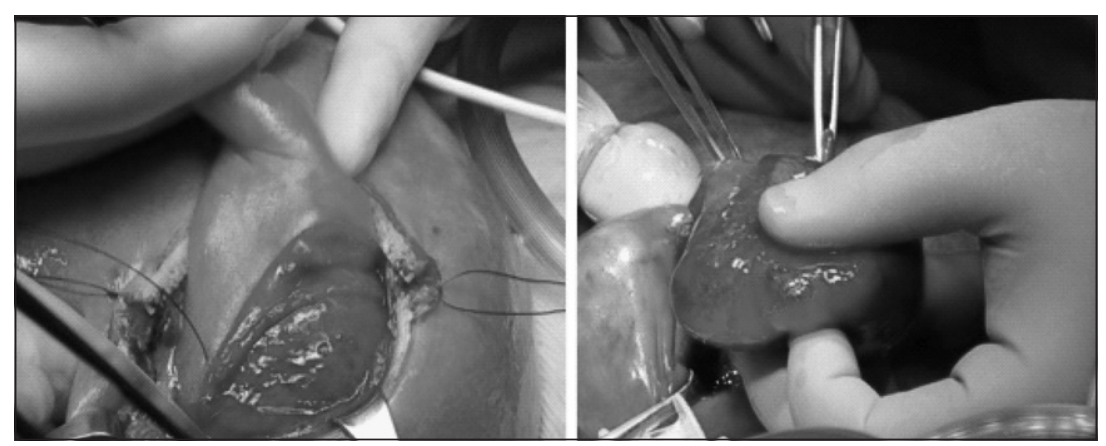

Figura 1. Cirugía fetal abierta para resección de un malformación congénita adenomatoidea pulmonar. Fotos del centro de cirugía fetal del Hospital de niños de Cincinnati, EE.UU., con permiso. 
exposición de los elementos neurales al ambiente intrauterino. El segundo, y es la que se cree que produce el mayor daño, estaría dado por la exposición prolongada del los elementos neurales a la irritación química del líquido amniótico y el trauma directo por roce contra la pared del útero.

Extensa investigación en modelos animales de MMC ha demostrado que la corrección temprana del defecto corrige la malformación de Arnold-Chiari tipo 2, previniendo así el desarrollo de hidrocefalia y disminución de la alteraciones motoras y vesicales ${ }^{20}$.

La primera corrección fetal en humanos de un MMC con éxito se realizó en 1998 en el hospital de niños de Filadelfia. En el 2011, se publicaron en el New England Journal of Medicine, los resultados del estudio randomizado y multicéntrico comparando los resultados del la corrección prenatal del MMC entre las semanas 19 y 25 de gestación (figura 2) y el manejo expectante. El estudio demostró una reducción significativa en la instalación de drenajes ventriculoperitoneales, de $82 \%$ en el grupo control a $40 \%$ en el grupo de cirugía prenatal. También demostró una mejoría importante en la función neuromotora, resultando en un $42 \%$ de independencia para caminar de los pacientes del grupo de cirugía fetal, comparado con $21 \%$ del grupo control. Además demostró una ausencia de herniación del cerebro posterior en $36 \%$ en el grupo de cirugía prenatal comparado con $4 \%$ en el grupo control ${ }^{19}$.

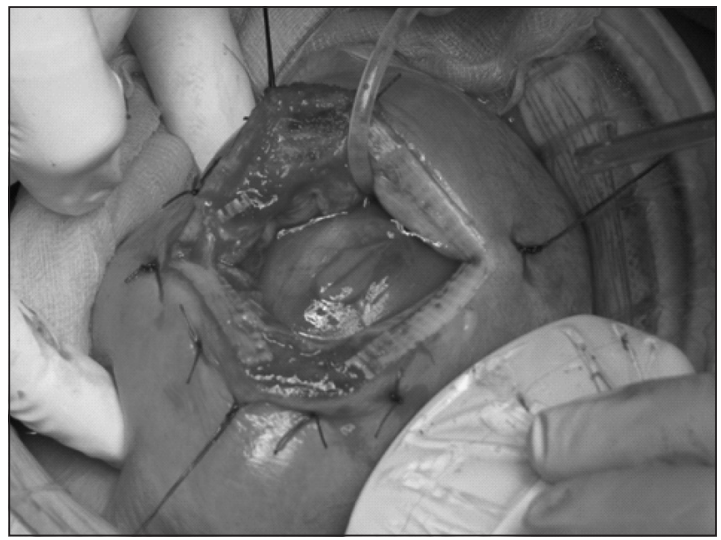

Figura 2. Cirugía fetal abierta para la corrección del mielomeningocele. Foto del centro de cirugía fetal. Hospital Vall d'Hebron, Barcelona, España. Con permiso.
A pesar de todos estos avances en el manejo prenatal del MMC, se continúa realizando investigación para poder desarrollar técnicas menos invasivas, tanto para la madre como para el feto, tratando de crear tejidos impermeables para cerrar el $\mathrm{MMC}^{21}$ y técnicas fetoscópicas para la reparación del defecto.

\section{Procedimiento Ex-utero Intrapartum Treatment (EXIT)}

Los avances en el diagnóstico prenatal han permitido detectar una serie de malformaciones congénitas que tienen un gran impacto en el periodo post natal inmediato, requiriendo estrategias para resolverlas inmediatamente antes de nacer.

El procedimiento de EXIT fue inicialmente descrito para revertir la oclusión traqueal al momento del nacimiento en fetos con hernia diafragmática severa que fueron tratados con oclusión traqueal con clips en útero para promover el crecimiento pulmonar ${ }^{22}$. Actualmente este procedimiento ha expandido su indicación a: 1) fetos con obstrucción de la vía aérea superior en que se anticipa un dificultad para obtener una vía aérea fácilmente (figura 3); 2) Fetos con masas mediastinicas o torácicas grandes que requieren de su resección para asegurar la ventilación; 3) situaciones en las cuales es necesario una intervención inmediata, ya que existe un riesgo elevado en transportar al neonato a un pabellón quirúrgico (tabla $3)$.

Dentro de las indicaciones de la vía aérea se incluyen masas cervicales en la región anterior, obstrucción congénita de la vía aérea alta, quistes sublinguales gigantes y el síndrome de hipoplasia craneofacial. Las masas torácicas están determinadas principalmente por grandes malformaciones congénitas adenomatoideas pulmonares con extensa desviación del mediastino o teratomas mediastínicos. Otras indicaciones son hernias diafragmáticas congénitas asociadas a cardiopatías congénitas que requieren conexión inmediata a la máquina de circulación extracorporea, o prematuros con teratomas sacrocoxigeos asociados a descompensación cardiovascular que requieren 

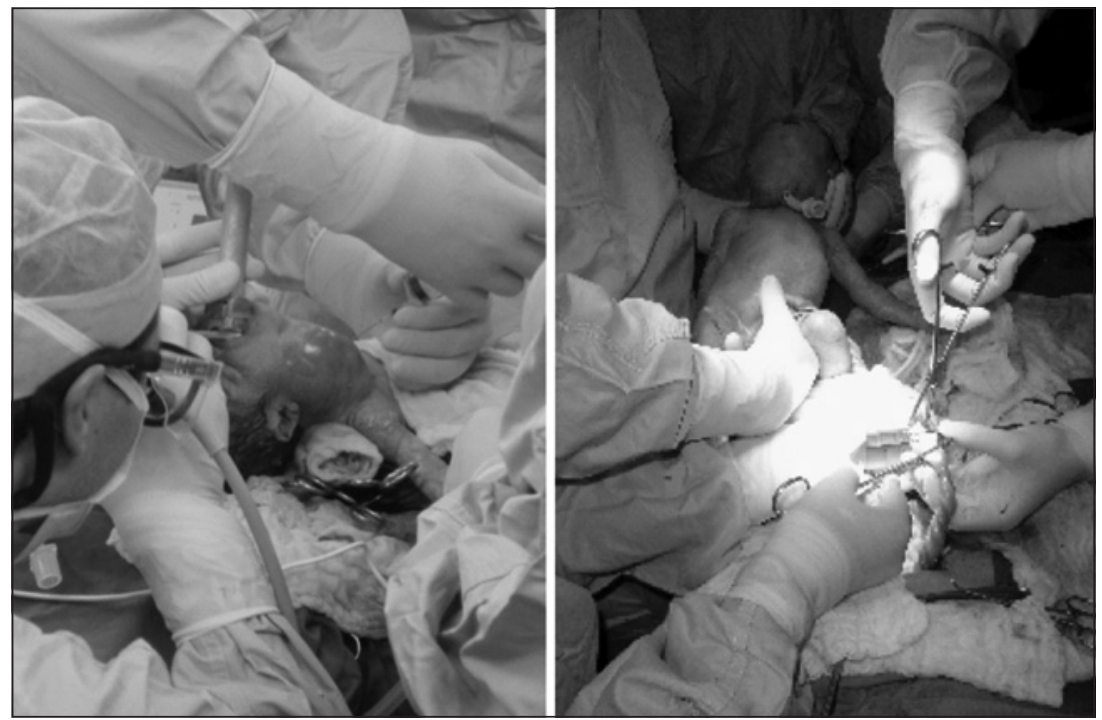

Figura 3. Procedimiento de EXIT en feto con tumor cervical. Fotos de la Unidad de Cirugía Pediátrica, Hospital Clínico, Pontificia Universidad Católica de Chile, con permiso.

Tabla 3. Indicaciones actuales para el procedimiento de EXIT

\begin{tabular}{|l|}
\hline Masas cervicales fetales \\
Teratomas cervicales \\
Hemangiomas \\
Gota \\
Neuroblastomas \\
Masas pulmonares \\
Malformaciones congénitas adenomatoideas pulmonares \\
Secuestros brocopulmonares \\
Masas mediastínicas \\
Teratomas \\
Linfangiomas \\
Síndrome de obstrucción congénita de la vía aérea alta \\
EXIT a ECMO \\
Hernia diafragmáticas asociadas a cardiopatias. \\
Teratomas sacrocoxigeos \\
\hline
\end{tabular}

la resección inmediata de la tumoración para poder estabilizarlo ${ }^{23,24}$.

La parte más importante del procedimiento de EXIT es la mantención del flujo útero-placentario. Esto se logra posicionando a la madre en posición supina e inclinada parcialmente a izquierda para optimizar el retorno venoso y maximizar el flujo sanguíneo al útero y placenta. Para conseguir esto, se usa una anestesia general profunda con agentes halogenados para alcanzar la relajación uterina necesaria mientras se mantiene el flujo materno cerca del basal. Otro punto importante es la anestesia fetal. Aunque los gases anestésicos pueden cruzar la placenta y proveer con suficiente anestesia al feto, se suplementa con dosis de agentes narcóticos y agentes paralíticos para prevenir el disconfort fetal y los esfuerzos respiratorios durante el procedimiento. Previo a la apertura del útero, es importante realizar un mapeo de la placenta para abrir el útero alejado de los bordes y así prevenir los desprendimientos agudos de placenta. Una vez abierto el útero, se debe colocar una vía periférica en el feto para resucitación con fluidos, transfusión de sangre y administración de drogas si lo requiriera. Un oxímetro de pulso de debe colocar en la mano del feto expuesta para monitorizar la oxigenación durante la cirugía, y se debe realizar la monitorización cardiaca del feto con un ecocardiograma en forma continua para evaluar la función cardíaca y el volumen circulatorio. Una infusión de Ringer lactato tibio se debe usar en el útero para mantener el volumen uterino y así prevenir compresiones del cordón umbilical. Se debe exponer lo menos posible el feto al ambiente, sólo exteriorizando la zona donde se realizará el procedimiento, de esta forma ayuda a mantener el volumen uterino y la temperatura del feto ${ }^{23,24}$. 
Debe existir una perfecta sincronización con el equipo anestésico durante el procedimiento. Una vez seccionado el cordón umbilical después de haber terminado el procedimiento en el feto, se deben cortar los gases anestésicos e iniciar la infusión de oxitocina para promover la contracción uterina. Los riesgos maternos de este procedimiento incluyen hemorragias, dehiscencia de la sutura uterina, ruptura uterina en los próximos embarazos e infección de la herida operatoria ${ }^{23,24}$.

El procedimiento de EXIT en general dura aproximadamente 60 minutos, pero están descritas hasta de 2,5 horas.

\section{Conclusiones}

La cirugía materno-fetal nace de la necesidad de un grupo de especialistas que no aceptaron la evolución natural de las gestaciones con defectos congénitos letales.

En los últimos 30 años, grandes avances se han hecho en el campo de la medicina fetal, logrando mejores imágenes del feto con malformaciones congénitas mediante ecografías o resonancias magnéticas, permitiendo entender con mayor exactitud su patofisiología. Importantes avances se han logrado en los métodos de tocolisis, especialmente en lo que se refiere a cirugía fetal abierta, permitiendo prolongar los embarazos intervenidos prenatalmente $\mathrm{y}$ así evitar agregar la morbilidad que implica por si sola la prematurez a un niño con un defecto congénito.

La medicina materno-fetal no puede ser realizada si no es por un equipo multidiciplinario, obstetras, neonatólogos, cirujanos pediatras, anestesistas, cardiólogos y muchas otras, que estén interesados y dedicados a esta disciplina de la medicina cada vez más especializada, que actúen dentro de protocolos estrictos de manejo, para así asegurar el bienestar tanto de la madre como del feto, y que cuenten con un apoyo de la institución donde trabajan ${ }^{25}$.

A pesar de los avances actuales, quedan muchos logros por venir. Desarrollar instrumentos más pequeños y refinados para realizar intervenciones endoscópicas intrauterinas, ya sea en el feto o en la placenta, que permitan visualizar el ambiente intrauterino con una gran nitidez y al mismo tiempo ser capaces de no producir alteraciones en la membranas que envuelven al feto, disminuyendo los riesgos de ruptura prematura de membranas. Otro foco de gran interés ha sido el desarrollo de líneas de investigación en el tratamiento de enfermedades genéticas y celulares, la cuales pueden ser tratadas en un futuro con células madres y terapia génica ${ }^{26,27}$, permitiendo ser menos invasivo con la madre y el feto.

\section{Agradecimiento}

Al Dr. Foong-Yen Lim del Centro de terapia fetal del Hospital de niños de Cincinnati. EEUU, por las fotografías del la toracotomía fetal. Al Dr. José Luis Peiró del Centro de terapia fetal del Hospital Vall d'Hebron, Barcelona, España, por la fotografía de la reparación fetal del MMC. Al Dr. Alejandro Zavala de la Unidad de Cirugía Pediátrica, División de Cirugía, Pontificia Universidad Católica de Chile, por la fotografía del procedimiento de EXIT.

\section{Referencias}

1.- Harrison MR, Golbus MS, Filly RA, et al: Fetal surgery for congenital hydronephrosis. N Engl J Med 1982; 306 : 591-3.

2.- Harrison MR, Filly RA, Golbus MS, et al: Fetal treatment 1982. Landmark consensus statement by fetal surgeons at the Kroc Foundation Symposium providing the first patient selection guidelines for fetal hydrocephalus and other congenital anomalies. N Engl J Med 1982; 307 (26): 1651-2.

3.- Azizkhan RG, Crombleholme TM: Congenital cystic lung disease: contemporary antenatal and postnatal management. Pediatr Surg Int 2008; 24: 643-57.

4.- Adzick N: Open fetal surgery for life-threatening fetal anomalies. Semin Fetal Neonat Med 2010; 15: 1-8.

5.- Witlox RS, Lopriore E, Oepkes D. Prenatal interventions for fetal lung lesions. Prenat Diagn 2011; 31: 628-36.

6.- MacGillivray TE, Harrison MR, Goldstein RB, et al: Disappearing fetal lung lesions. J Pediatr Surg 1993; 28 : 1321-4. 
7.- Davenport M, Warne SA, Cacciaguerra S, et al: Current outcome of antenally diagnosed cystic lung disease. J Pediatr Surg 2004; 39: 549-56.

8.- Thorpe-Beeston JG, Nicolaides KH: Cystic adenomatoid malformation of the lung: prenatal diagnosis and outcome. Prenat Diagn 1994; 14: 677-88.

9.- Crombleholme TM, Coleman B, Hedrick H, et al: Cystic adenomatoid malformation volume ratio predicts outcome in prenatally diagnosed cystic adenomatoid malformation of the lung. J Pediatr Surg 2002; 37: 3318.

10.- Adzick NS, Harrison MR, Glick PL, Golbus MS, et al: Fetal cystic adenomatoid malformation: prenatal diagnosis and natural history. J Pediatr Surg 1985; 20: 483-8.

11.- Adzick NS, Harrison MR, Crombleholme TM, Flake AW, et al: Fetal lung lesions: management and outcome. Am J Obstet Gynecol 1998; 179: 884-9.

12.- Tsao K, Hawgood S, Vu L, et al: Resolution of hydrops fetalis in congenital cystic adenomatoid malformation after prenatal steroid therapy. J Pediatr Surg 2003; 38 : 508-10.

13.- Wilson RD, Baxter JK, Johnson MP, King M, et al: Thoracoamniotic shunts: fetal treatment of pleural effusions and congenital cystic adenomatoid malformation. Fetal Diagn Ther 2004; 19: 413-20.

14.- Hedrick HL, Flake AW, Crombleholme TM, Howell LJ et al: The exutero intrapartum therapy procedure for highrisk fetal lung lesions. J Pediatr Surg 2005; 40: 1038-43 discussion 1044.

15.- Adzick NS: Fetal myelomeningocele: natural history, pathophysiology, and in-utero intervention. Semin Fetal Neonatal Med 2011; 15: 9-14.

16.- Thompson D: Postnatal management and outcome for neural tube defects including spinda bifida and encepha- loceles. Prenat Diagn 2009; 29: 412-9.

17.- Tuli S, Drake J, Lamberti-Pasculli M: Long-term outcome of hydrocephalus management in myelomeningoceles. Childs Nerv Syst 2003; 19: 286-91.

18.- Bowman RM, McLone DG, Grant JA, et al: Spina bifida outcome: a 25-year prospective. Pediatr Neurosurg 2001; 34: 114-20.

19.- Adzick NS, Thom EA, Spong CY, et al: A randomized trial of prenatal versus postnatal repair of Myelomeningocele. N Engl J Med 2011; 364: 993-1004.

20.- Meuli M, Meuli-Simmen C, Hutchins GM, et al: In utero surgery rescues neurologic function at birth in sheep with spina bifida. Nat Med 1995; 1: 342-7.

21.- Fontecha CG, Peiro JL, Aguirre $M$, et al: Inert patch with bioadhesive for gentle fetal surgery of myelomeningocele in a sheep model. Eur J Obstet Gynecol Reprod Biol 2009; 146 (2): 174-9.

22.- Mychalishka GB, Bealor JF, Graf JL, et al: Operating on placental support: the ex utero intrapartum treatment (EXIT) procedure. J Pediatr Surg 1997; 32: 22-30.

23.- Liechty K. Ex-utero intrapartum therapy. Semin Fetal Neonat Med 2010; 15: 34-9.

24.- Marwan A, Crombleholme TM: The EXIT procedure: principles, pitfalls, and progress. Semin Pediatr Surg. 2006; 15: 107-15.

25.- Shue EH, Harrison M, Hirose S: Maternal-fetal surgery: history and general considerations. Clin Perinatol 2012; 39 (2): 269-78.

26.- Nijagal A, Flake AW, MacKenzie TC. In utero hematopoietic cell transplantation for treatment of congenital anomalies. Clinics in Perinatology; 39 (2): 301-10. Epub 2012 May 8.

27.- Santore MT, Roybal JL, Flake AW: Prenatal stem cell transplantation and gene therapy. Clin Perinatol. 2009; 36: 451-71. 